\title{
Client Puzzles: Effective Defence against Resource Inflation Threats
}

\author{
Smita M. Miraje ${ }^{1}$, Manisha Bharti ${ }^{2}$ \\ ${ }^{1}$ Savitribai Phule Pune University, Indira College of Engineering and Management, Pune, Maharashtra, India \\ ${ }^{2}$ Professor, Savitribai Phule Pune University, Indira College of Engineering and Management, Pune, Maharashtra, India
}

\begin{abstract}
DoS and DDoS are among the real dangers to digital security, and customer riddle, which requests a customer to perform computationally costly operations before being allowed administrations from a server, is an understood countermeasure to them. On the other hand, an assailant can inflate its capacity of DoS/DDoS assaults with quick astound illuminating programming and/or inherent graphics handling unit (GPU) equipment to significantly debilitate the adequacy of customer riddles. In this paper, we concentrate how to counteract DoS/DDoS aggressors from inflating their riddle illuminating capacities. To this end, we present another customer riddle alluded to as programming riddle. Dissimilar to the current customer riddle plans, which distribute their riddle calculations ahead of time, a riddle calculation in the present programming riddle plan is arbitrarily created strictly when a customer solicitation is gotten at the server side and the calculation is produced such that: an aggressor can't set up an execution to illuminate the riddle ahead of time and the assailant needs significant exertion in deciphering a focal handling unit riddle programming to its practically equal GPU form such that the interpretation is impossible progressively. Also, we demonstrate to execute programming riddle in the bland serverprogram.
\end{abstract}

Keywords: Software puzzle, code obfuscation, GPU programming, distributed denial of service (DDoS).

\section{Introduction}

DoS and DDoS are viable if aggressors spend a great deal less assets than the casualty server or are a great deal more intense than ordinary clients. In the case over, the aggressor spends immaterial exertion in creating a solicitation, however the server needs to spend a great deal more computational exertion in HTTPS handshake (e.g., for RSA unscrambling). For this situation, ordinary crypto-realistic apparatuses don't upgrade the accessibility of the administrations; truth be told, they may debase administration quality because of costly cryptographic operations. The reality of the DoS/DDoS issue and their expanded recurrence has prompted the coming of various guard systems.

In this paper, we are especially keen on the countermeasures to DoS/DDoS assaults on server calculation power. Let $\gamma$ signify the proportion of asset utilization by a customer and a server. Clearly, a countermeasure to DoS and DDoS is to expand the proportion $\gamma$, i.e., build the computational expense of the customer or diminishing that of the server. User riddle is a surely understood way to deal with expansion the expense of customers as it strengths the customers to complete substantial operations before being conceded administrations. By and large, a customer riddle plan comprises of three stages: riddle generation, puzzle tackling by the customer and riddle verification by the server.

\section{Problem Specification}

DoS and DDoS are viable if assailants spend substantially less assets than the casualty server or are considerably more capable than ordinary clients. The assailant spends unimportant exertion in creating a solicitation, however the server needs to spend a great deal more computational exertion in HTTPS handshake (e.g., for RSA decoding). For this situation, routine cryptographic instruments don't improve the accessibility of the administrations; truth be told, they may corrupt administration quality because of costly cryptographic operations.[1][2][3].

\section{Related Work}

A Graph Approach to Quantitative Analysis ofControlFlow Obfuscating Transformations

Advanced jumbling systems are expected to dishearten figuring out and pernicious altering of programming projects. We study control-flow confusion, which works by altering the control flow of the system to be jumbled, and watch that it is difficult to assess the heartiness of these obscurity strategies. In this paper, we display a structure for quantitative examination of control-flow muddling changes. Our structure is based upon the control-flow diagram of the system, and we demonstrate that numerous current controlflow confusion strategies can be communicated as an arrangement of essential changes on these charts. We likewise propose another measure of the difficulty of switching these jumbled projects.[1]

\section{Acceleration of AES encryption on CUDA GPU}

GPU displays the capacity for applications with an abnormal state of parallelism in spite of its ease. The backing of whole number and consistent guidelines by the most recent era of GPUs empowers us to execute figure calculations all the more effortlessly. Nonetheless, choices, for example, parallel handling granularity and memory allotment force a substantial weight on developers. [2]

Language-Independent Sandboxing of Just-In-Time Compilation and Self-Modifying Code

At the point when managing dynamic, untrusted substance, for example, on the Web, programming conduct must be 


\section{International Journal of Science and Research (IJSR) \\ ISSN (Online): 2319-7064 \\ Index Copernicus Value (2013): 6.14 | Impact Factor (2014): 5.611}

sandboxed, ordinarily through utilization of a dialect like JavaScript. Be that as it may, notwithstanding for such uncommonly composed dialects, it is difficult to guarantee the security of exceedingly improved, dynamic dialect runtimes which, for efficiency, depend on cutting edge strategies, for example, Just-In-Time (JIT) arrangement, expansive libraries of local code bolster schedules, and many-sided systems for multi-threading and junk gathering. Each new runtime gives another potential assault surface and this security danger raises an obstruction to the selection of new dialects for making untrusted content. Uprooting this confinement, this paper presents general instruments for securely and efficiently sandboxing programming, for example, dynamic dialect runtimes, that make utilization of cutting edge, low-level procedures like runtime code modification. Our language in dependent sandboxing expands on Software-based Fault Isolation (SFI), a generally static system. We give a more flexible type of SFI by including new requirements and instruments that permit security to be ensured in spite of runtime code modifications. We have added our augmentations to both the x86-32 and x86-64 variations of a creation quality, SFIbased sandboxing stage; on those two architectures SFI components face diverse difficulties. We have likewise ported two agent dialect stages to our broadened sandbox: the Mono basic dialect runtime and the V8 JavaScript motor. In point by point assessments, we find that sandboxing lull changes between distinctive benchmarks, dialects, and equipment stages.[3]

\section{Mitigating Bandwidth-Exhaustion Attacks using Congestion Puzzles}

We display blockage astounds (CP), another countermeasure to data transfer capacity fatigue assaults. Like different resistances taking into account customer riddles, CP endeavors to drive assailants to put inconceivable assets keeping in mind the end goal to effectively perform denialof-administration assaults. Not at all like past riddle based methodologies, on the other hand, our own is the first intended for the transfer speed depletion assaults that are normal at the system (IP) layer. At the center of CP is an exquisite circulated riddle component that licenses switches to agreeably force and check confounds. We exhibit through examination and reenactment that $\mathrm{CP}$ can effectively guard systems from flooding assaults without depending on the definition of assault marks to filter traffic. Besides, the same number of such assaults are directed by "zombie" PCs that have been quietly seized without the learning of their proprietors, the overheads that $\mathrm{CP}$ forces on intensely connected with zombies can improve the probability that the PC's proprietor identifies the bargain and makes a move.[4]

\section{Proofs of Work and Bread Pudding Protocols(Extended Abstract)}

We formalize the thought of a proof of work (POW). In numerous cryptographic conventions, a prover tries to persuade a verifier that she has information of a mystery or that a certain scientific connection remains constant. By complexity, in a POW, a prover exhibits to a verifier that she has performed a sure measure of computational work in a predetermined interim of time. POWs have served as the premise of various security conventions in the writing, however have up to this point needed cautious portrayal. In this paper, we offer definitions treating the thought of a POW and related ideas. We likewise present the needy thought of a bread pudding convention. Bread pudding is a dish that started with the motivation behind reusing bread that has gone stale. In the same soul, we characterize a bread pudding convention to be a POW such that the computational exertion put resources into the evidence may be reused by the verifier to accomplish a different, valuable, and obviously revise calculation. As a sample of a bread pudding convention, we demonstrate how the MicroMint plan of Rivest and Shamir can be separated into a gathering of POWs. These POWs can not just serve in their own particular perfectly fine for security conventions, however can likewise be reaped so as to outsource the MicroMint stamping operation to an expansive gathering of untrusted computational gadgets.[5]

\section{pTCP: A Client Puzzle Protocol For Defending Against ResourceExhaustion Denial of Service Attacks}

In the course of recent years, foreswearing of administration (DoS) assaults have turned out to be all the more a danger than any time in recent memory. DoS assaults are gone for denying or exhausting so as to debase administration for an authentic client the assets for a specific framework. Customer riddle conventions have gotten consideration as of late as a strategy for battling DoS assaults. In a customer riddle convention, the customer is compelled to comprehend a cryptographic riddle before it can set up an association with a remote server. This paper presents a novel customer riddle convention that uses a change of the Extended Tiny Encryption Algorithm. A usage of the customer riddle convention was finished in the TCP pile of the Mandrake Linux 9.2 working framework. We call this change to the TCP stack pTCP (for Puzzle TCP). Our customer riddle calculation is quick, and is compact to different frameworks and architectures. All the more significantly, it is extremely compelling against association consumption DoS assaults and other asset weariness DoS assaults (on the server) in light of the fact that negligible calculation burden is forced on the server to confirm the answer for a given riddle. Our customer riddle convention is likewise compelling against different other asset weariness assaults inside of the vehicle layer, and can avert assaults that exist at the application layer. In this paper, we depict our customer riddle convention in subtle element, and demonstrate its adequacy against DoS assaults by utilizing trial results.[6]

\section{Reconstructing Hash Reversal based Proof of Work Schemes}

Evidence of work plans use customer riddles to oversee constrained assets on a server and give strength to dissent of administration assaults. Assaults using GPUs to inflate computational limit, known as asset inflation, are a novel and capable risk that significantly build the computational divergence between customers. This dissimilarity renders confirmation of work plans in view of hash inversion insufficient what's more, possibly damaging. This paper looks at different such plans in perspective of GPU-based assaults and identifies attributes that permit barrier systems to withstand assaults. Specifically, we exhibit that, hashinversion plans which adjust exclusively on server burden are ineffectual under assault by GPU using foes; while.[7]

\section{Volume 4 Issue 12, December 2015}




\section{International Journal of Science and Research (IJSR) \\ ISSN (Online): 2319-7064}

Index Copernicus Value (2013): 6.14 | Impact Factor (2014): 5.611

\section{Resource Inflation Threats toDenial of Service Countermeasures}

Money based instruments have been proposed as an approach to utilize asset decency among contenders or a support of foil Denial of Service (DoS) assaults. Under asset reasonableness, a server assigns its support of the customers in extent to their installment of an asset, making the asset serve as a sort of coin. We consider the defenselessness of money based DoS safeguard instruments to different asset inflation assaults in which an aggressor can significantly inflate its ownership of the asset with ease and in a manner that may be either difficult or undesirable for a substantial customer to do. We give a basic hypothetical examination of asset inflation assaults and research its application to various installment plans to rank their presumable powerlessness. We find that the risk of Graphics Processing Units (GPUs) for inflation assaults is particularly extreme: we have the capacity to show inflation of up to $630 \mathrm{x}$ with basic cheap GPUs. We additionally audit dangers from different abilities, including multi-center processors, distributed computing.[8].

\section{Time-lock Puzzles and Timed-release Crypto}

Our inspiration is the thought of "timed-discharge crypto,"'" where the objective is to scramble a message with the goal that it can not be unscrambled by anybody, not even the sender, until a pre-decided measure of time has passed. The objective is to "send data into the future."'" This issue was initially talked about by Timothy May lcite\{May93\}. What are the uses of "timed-discharge crypto"'"? Here are a couple of potential outcomes (some because of May): A bidder in a bartering needs to seal his offer with the goal that it must be opened after the offering period is shut. A property holder needs to give his home loan holder a progression of encoded home loan installments. These may be encoded advanced money with distinctive unscrambling dates, so that one installment gets to be decryptable (and along these lines usable by the bank) toward the start of each progressive month. An individual needs to encode his journals with the goal that they are just decryptable following fifty years. A key-escrow plan can be founded on timed-discharge crypto, so that the administration can get the message keys, yet strictly when a settled period (say one year). $\backslash$ There are probably numerous different applications. There are two regular ways to deal with executing timed-discharge crypto: Use "time-lock puzzles"'"- - computational issues that can not be tackled without running a PC constantly for no less than a sure measure of time. Use trusted operators who guarantee not to uncover certain data until a predetermined date. Utilizing trusted specialists has the conspicuous issue of guaranteeing that the operators are dependable; mystery sharing methodologies can be utilized to ease this worry. Utilizing time-lock riddles has the issue that the CPU time required to take care of an issue can rely on upon the sum and nature of the equipment used to take care of the issue, and also the parallelizability of the computational issue being fathomed. In this note we investigate both methodologies. (We take note of that Tim May has recommended a methodology in view of the utilization of trusted operators.)[9].

\section{Proposed Methodology}

A Proposed Structure has a software perplex, the server needs to execute three modules: Puzzle Core Generation, Puzzle Challenge Generation, Code Protection, as appeared in figure

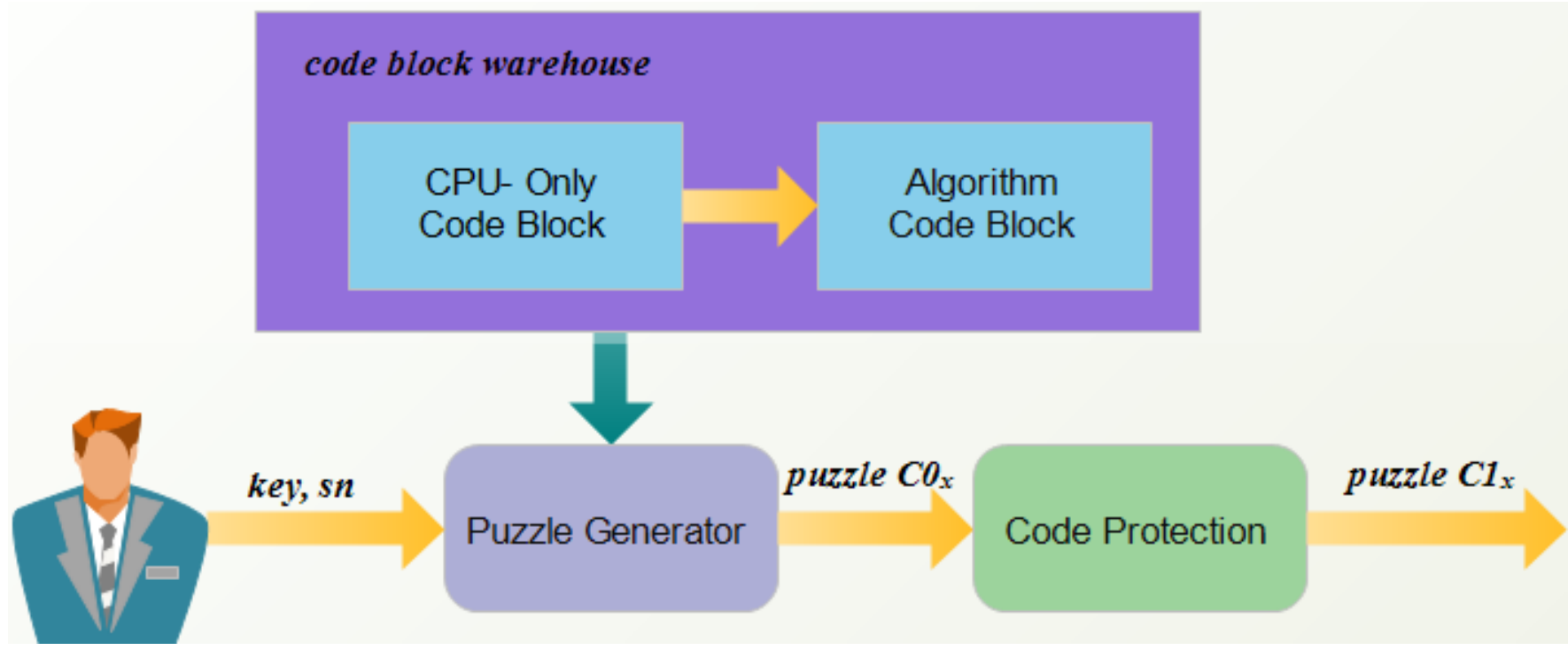

1) Puzzle Core Generation: From the code piece product house, the server first picks $\mathrm{n}$ code squares taking into account hash capacities and a mystery, e.g., the jth guideline square $b$. and afterward it creates the Puzzle cente

2) Puzzle Challenge Generation: by Given some helper info messages, for example, IP addresses, and in-line constants, the server ascertains a message $\mathrm{m}$ from open information, for example, their IP locations, port numbers and treats, and creates a test, like scrambling plaintext.

3) Code Protection: Instinctively, code jumbling has the capacity obstruct the above interpretation risk to some degree. In spite of the fact that there are no nonexclusive muddling procedures which can keep a patient and propelled programmer from comprehension a project in

\section{Volume 4 Issue 12, December 2015}


principle, results in demonstrate that jumbling build the expense of figuring out. Accordingly, despite the fact that code muddling may be not agreeable in long haul software barrier against hacking, it is suitable for strengthening software bewilders which request an insurance time of a few seconds just.

A software puzzle comprises of teaches, and every guideline has a structure (opCode, [operands]), where opCode shows which operation (e.g., expansion, movement, bounce) is, while the operands, differing with opCode, are the parameters (e.g., target location of hop direction) to finish the operations. As a prevalent muddling innovation, code encryption innovation regards software code as information string and scrambles both operand and opCode.

When a software puzzle is made at the server side, it will be conveyed to the customer who demands for services over a frail channel, for example, Web, and keep running at the customer's side.[6][8].

\section{Conclusion}

In this paper we considered the dangers from a scope of assaults against different coin based DoS countermeasures. Specifically, we presented the idea of asset inflation assaults on coin based DoS countermeasures in which the aggressors find approaches to inflate their responsibility for asset (installment) required for getting administration. As a contextual analysis, we showed a progression of asset inflation assaults on existing DoS systems. Case in point, our investigations with asset inflation assaults on riddle based plans demonstrated that an aggressor can utilize multi-center processors, distributed computing, and GPUs to inflate its assets. Specifically, the asset inflation assault utilizing GPUs demonstrated to make an impressive inflation component of up to $600 x$ utilizing

\section{References}

[1] A Graph Approach to Quantitative Analysis of Control-Flow Obfuscating Transformations.

[2] Acceleration of AES encryption on CUDA GPU.

[3] Language-Independent Sandboxing of Just-In-Time Compilation and Self-Modifying Code.

[4] Mitigating Bandwidth-Exhaustion Attacks using Congestion Puzzles.

[5] Proofs of Work and Bread Pudding Protocols(Extended Abstract).

[6] pTCP: A Client Puzzle Protocol For Defending Against Resource Exhaustion Denial of Service Attacks.

[7] Reconstructing Hash Reversal based Proof of Work Schemes.

[8] Resource Inflation Threats to Denial of Service Countermeasures.

[9] Time-lock Puzzles and Timed-release Crypto. 